\title{
CHALLENGES IN WIRELESS SENSOR NETWORKS - OVERVIEW
}

\section{Vasilije Radović , Nebojša Bačanin, Miodrag Živković}

\section{Singidunum University,} Belgrade, Serbia

\begin{abstract}
:
Wireless sensor networks can be found almost everywhere. They consist of small devices, known as sensor nodes, which are arranged in the desired environment. They collect information and send it to the client through a network gateway using a routing protocol. These networks have many good characteristics like quality of service (QoS), reliability, low power consumption, flexibility, scalability, etc. Despite these good characteristics, many problems and challenges are plaguing these networks. Challenges depend on the type of network, and each network has its problems. Connecting wireless sensor networks to the Internet of Things also leads to new challenges. Security is one of the main problems plaguing these networks, but it is also one of the most difficult challenges due to network limitations. In addition to safety, the biggest challenge is still consuming energy because it is not always possible to charge the battery, so the sensor nodes die. Another more serious challenge is the real-time transmission. Traditional wireless sensor networks do not work in real-time, and even if they need to work in real-time, they avoid it. The Internet of Things works in real-time, so it needs real-time wireless sensor networks.
\end{abstract}

\section{Keywords:}

Wireless sensor networks, Sensor node, Challenge, Issues, Characteristics, Internet of Things, Energy, Security.

\section{INTRODUCTION}

Wireless sensor networks are one of the most important technologies of this century. These networks are applicable in almost all areas of human activity. Because of their great potential, those networks are of considerable help in so many fields. The most important fields of application are military, health, environment, industry, traffic, ecology, etc. The main advantages of wireless sensor networks are low cost and low energy consumption, which explains such widespread application. The role of wireless sensor networks is to track or monitor the environment, in which they succeed through collection, processing, and transmission of data. Wireless sensor networks ensure their survival in the Internet of Things, as they are the main component of the Internet of Things. Smart cities, smart homes, and others are being developed with the help of the Internet of Things.
Correspondence:

Miodrag Živković

e-mail:

mzivkovic@singidunum.ac.rs 
These developments are where the sensor networks play their main role. Nowadays, sensor networks can connect to the internet. But these networks still have many problems and flaws, making them so challenging that we are constantly working on improvement. Despite all the research that has been conducted on how to avoid the limitations of these networks, there are still too many challenges and issues in this field. There are challenges in all types of networks, network characteristics, design, architecture, protocols, security, Internet of Things, etc.

\section{WIRELESS SENSOR NETWORKS}

A wireless sensor network is a large group of sensor nodes. This network consists of sensor nodes, gateway, communication protocols, and client. Sensor nodes can collect and process data and then send the data to other nodes until it reaches the gateway. The task of the gateway is to send data to the client. Communication protocols organize data transfer and ensure that data arrives at its destination.

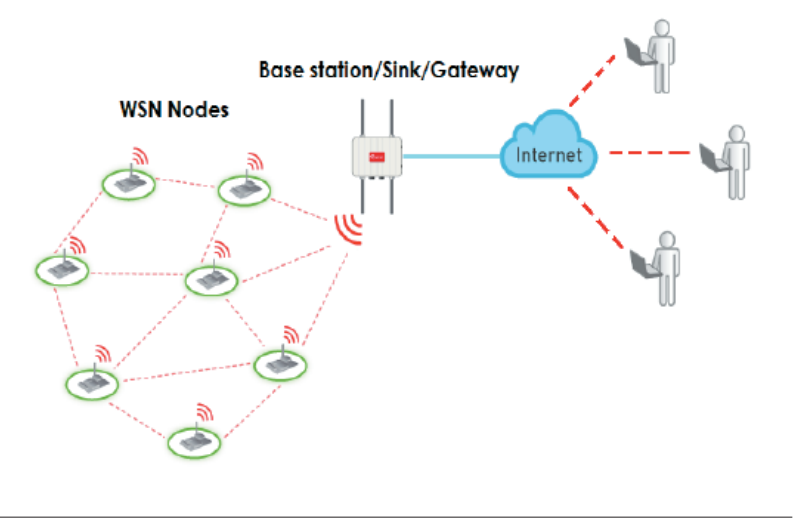

Figure 1 - Wireless sensor network

Wireless sensor networks usually don't have any infrastructure. Many sensor nodes are randomly placed to monitor the environment without an infrastructure, which is called an unstructured network. In unstructured networks, sensor nodes can self-organize. In structured networks, the seniors are placed according to a previously devise plan.

\subsection{SENSOR NODE}

The main components of the sensor node are:

- sensor

- microcontroller

- transceiver

- memory

- power supply

Sensors collect data, the microcontroller processes the data, and the transceiver forwards or receives data from other nodes. The memory is used to store data, and the power supply is a battery. Sensor nodes are small and limited, which is why they are cheap [1]. Yet, they know how to be energy efficient because the batteries are small.

Optional components of the sensor node are a GPS location receiver and a power generator if the sensor needs more power.

\subsection{CHARACTERISTICS}

For wireless sensor networks to be well implemented, the best possible characteristics are needed. It is necessary to make a lot of compromises for a better network, although with better quality comes a higher price. The characteristics of these networks are:

- Robustness

- Low price

- Reliability

- Mobility

- Dynamic network organization

- Low energy consumption

- Network heterogeneity

- Quality of service (QoS)

- Real-time transmission

- Flexibility

- Scalability

These characteristics are a good fit for applications in various areas. Still, wireless sensor networks have so many issues and challenges. Among the challenges is the improvement of the main characteristics of these networks. 


\section{CHALLENGES}

Sensor networks have advanced a lot, but there are still issues and challenges for researchers and scientists. Figure 2 shows the challenges that are now being considered or have yet to be considered by researchers.

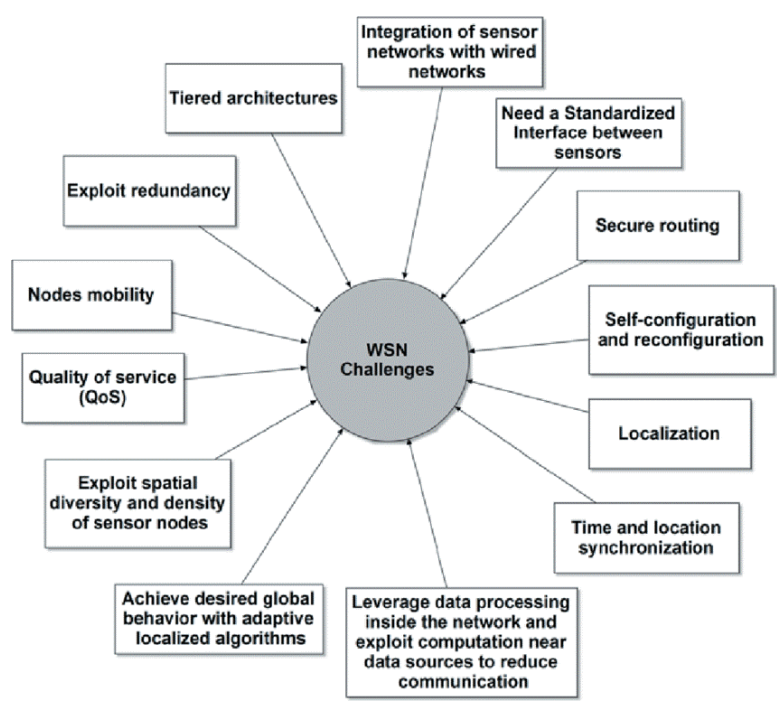

Figure 2 - Challenges in wireless sensor networks

This picture shows some challenges, although of course, there are many more. The most important challenges are real-time transmission without delay, power management with good energy utilization, more secure and private protocols, high quality of service, scalability, heterogeneity, complexity, and better self-management. A large number of proposed architectures emerged recently that target some of the mentioned challenges. For instance, energy-efficient architecture that also incorporates security aspects is proposed in [2].

Generally speaking, challenges in wireless sensor networks can be divided based on network type, network components, network characteristics, the Internet of Things applications, and security [3], [4].

\subsection{CHALLENGES BY NETWORK TYPE}

Wireless sensor networks are installed everywhere, both underwater and underground, as well as on land. Every environment has its challenges [5] [6].

Types of wireless sensor networks are:

- Terrestrial

- Underground
- Underwater

- Multi-media

- Mobile

Terrestrial wireless sensor networks can have a significant number of sensor nodes. The nodes can be deployed in two ways in the area. The first way is ad hoc, where sensor nodes are randomly dropped in the area. The second deployment way is according to the previous plan, for example, using optimal placement. The main challenge in those networks is energy saving. To overcome that challenge, we can minimize delays, eliminate data redundancy, use multi-hop optimal routing, and use a short transmission range.

Underground wireless sensor networks are located underground to monitor caves, mines, or other activities. For the data to reach its destination, the gateway is above ground, which ensures that the data will reach the user. The main challenges are communication and energy consumption. In communication, signal attenuations occur, and often the signal is lost completely. There are problems with energy consumption because there is no way to recharge the batteries. More batteries are installed, but when the batteries run out, the sensor node dies. To solve these challenges, minimal energy consumption and the best possible communication protocols are necessary.

Underwater wireless sensor networks are much more expensive than conventional terrestrial networks. Fewer sensor nodes are set up, and underwater vehicles are used for information gathering and research. Acoustic waves are used for communication. Communication challenges include signal loss, limited bandwidth, and high delay. Preventing sensor node failure due to water conditions is the biggest challenge of these networks, they must be well equipped for the environmental conditions.

Multi-Media Wireless Sensor Networks are used to monitor multimedia content such as video, images, and audio. In these networks, the sensor nodes consist of cameras and microphones.

They are set according to previously conceived plans. The main challenges are power consumption, data processing, quality of service (QoS), the need for high bandwidth, compression, and cross-layer design. High bandwidth and high-power consumption are required for video transmission. The quality of service (QoS) is difficult to achieve due to delays [7]. 
Mobile wireless sensor networks consist of movable sensor nodes. Sensor nodes change their location and organize themselves. A node can only communicate with nodes within its range. These sensor nodes use dynamic routing protocols. The main challenges are coverage of the required area, energy consumption, control and navigation, localization, placement, data processing, and self-organization.

\subsection{CHALLENGES BY NETWORK COMPONENTS}

The main challenges associated with wireless sensor network components are their low cost and the fact that they are as small as possible. Sensor nodes are the most important components of these networks.

The challenges of sensor nodes are:

- Node costs

- Design constraints

- Deployment

- Energy consumption

- Fault-Tolerance

Since wireless sensor networks are usually huge, the price of one sensor node must be as low as possible. The goal is for the price to be less than one dollar. The low cost of nodes ensures the greater application of sensor networks as in that case they are not financially demanding.

When designing a sensor node, the goal is to make small devices that are cheap and efficient. As a result, software and hardware limitations arise. Hardware is limited by price and size, and then the software is limited by hardware components. A lot of compromises are needed to get efficient sensor nodes. Hardware constraints also affect the protocols and algorithms that can be used. Due to the small memory, the software must be limited to storing only truly important information. The software has advanced a lot and manages to deal with hardware shortcomings, but a great number of improvements are still needed. The most difficult challenges are with the hardware.

Deployment of sensor nodes is a crucial challenge. Network complexity, as well as some other problems, can be reduced by a good deployment scheme. A huge number of sensor nodes are always placed in these networks, so it is necessary to use valid techniques. There are two types of deployment of these networks: dynamic and static deployments.
In static deployment, the sensor nodes are placed in predetermined locations. Dynamic or Ad Hoc deployment is used with mobile sensor nodes, as they organize themselves. Sensor nodes place them in the best possible locations, but they often change location due to network needs and environmental conditions. These sensor nodes must be able to do everything themselves, without consuming too much energy.

Energy consumption is one of the most important challenges with sensor nodes because battery life most often determines the life of a sensor node. Some sensor nodes can charge their batteries, and some can even replace the battery. However, the most common case is where the node dies when the battery is discharged, so the sensor node must save its battery as much as possible. The development of smaller batteries with a larger capacity is going very slow, so it is necessary to find other solutions to maintain the sensor node. Some sensor nodes use several different modes of operation depending on whether they transmit information or not. If they do not transmit data, it means that they are in sleep mode, which is how they save the battery.

Fault tolerance is a very complicated challenge. The sensor node can fail quite easily in the event of any damage or low battery. It is necessary to improve the sensor nodes to cooperate better with failures, but this is not an easy task. In the event of a sensor node failure, the network must continue to operate smoothly, which is why the network needs anti-failure techniques to be maintained. Fault-adapted protocols are also needed.

\subsection{CHALLENGES BY NETWORK CHARACTERISTICS}

Wireless sensor networks have many characteristics, as we saw in Chapter 2.2. Each of these characteristics is challenging and needs to be improved. The main challenges of this type are real-time transmission, quality of service (QoS), scalability, heterogeneity, and power management.

Real-time transmission is the transfer of data in realtime with time constraints on when the data is due to arrive. Small delays are allowed, and the data must arrive before the deadline. This transmission may cause more delays or data loss. The problem is that not enough attention has been paid to this challenge by researchers and protocols have not been developed for real-time transmission. The development of real-time transmission protocols is just beginning. Ordinary protocols either ignore real-time or send data as quickly as possible so they may arrive on time. 
This characteristic of wireless sensor networks is very important for the future of these networks and therefore a lot of attention must be paid to this challenge.

Many new challenges and problems will only arrive when it comes to real-time transmission development.

Quality of service (QoS) is very important for sensitive data, so this challenge is of great importance for wireless sensor networks. Therefore, the quality of service parameters must match the transmission requirements. If the parameters are bad, the data will not arrive at the destination at the agreed time and they will be lost. The RAP and SPEED protocols have been proposed to improve the quality of real-time services. The RAP protocol measures the required packet transmission rate based on the path and deadline. However, there is no guarantee that the package will arrive on time. The SPEED protocol controls the delay in the nodes based on the feedback, but even here we have no guarantee due to various interferences that can occur.

Scalability is the ability to check the number of sensor nodes without affecting the network. This is a very challenging task, as with the increase of the network the number of problems and failures increases, but also because the cost of maintaining the network is higher. Protocols are then prone to major errors, so work needs to be done to improve the protocol. For the network to withstand the addition of more nodes, we need fault protection techniques and better transmission control.

Heterogeneity is the ability to connect different types of sensor nodes within a single network. Different interfaces between different sensor nodes are required. Heterogeneous wireless sensor networks can be installed in different applications thanks to the variety of sensor nodes, which is what makes these networks quite powerful. Of course, there are many challenges with these networks due to the need for complex protocols and better characteristics like robustness and flexibility.

Power management is a challenge that can be solved by intelligent use of energy and power. To achieve this, we need efficient use of energy, efficient collection of energy, as well as storage and conversion of energy. Network performance must not suffer from power management. One solution is to use ultra-small chips that consume little energy. Another solution is to collect energy. Collecting energy is a big challenge because each type of energy is collected differently. Energy collection can be divided into bursters and ticklers. An example of bursters energy collection is a micro-generator, and an example of ticklers energy collection is a solar cell.
Recently, a popular approach is the application of nature-inspired approaches to tackle some of the challenges imposed by the WSN. These challenges include localization of the sensor nodes [8], optimization and prolonging the network lifetime [9], [10] , and energy efficiency [11].

\subsection{CHALLENGES OF THE INTERNET OF THINGS}

For the Internet of Things to function properly, a lot of improvement of wireless sensor networks is required. Many challenges arise with the advent of the Internet of Things applications. The various changes are in architecture, in how to advance access for more devices, high real-time transmission, and semantic representation and processing.

Wireless sensor networks are the infrastructure of the Internet of Things [12]. The changes in the network architecture are needed to adapt to new tasks. There are many differences in the Internet of Things applications, so there is no single architectural solution. There is also the problem of connecting different architectures if there is a need for them. It is necessary to work on this challenge to improve architectures and facilitate their use.

The number of devices connected to wireless sensor networks and the Internet of Things is constantly growing. Due to the increase in the number of devices in the networks, it is necessary to develop networks that can withstand the constant addition of new devices. Therefore, it is necessary to improve routing protocols, as well as to improve network access. When a new device is connected to the network, it is necessary to gain access to the network to be able to exchange information. There are scheduled-based and contention-based access technologies.

The Internet of Things works in real-time, which is why wireless sensor networks must transmit in realtime. Since traditional sensor networks do not work in real-time or avoid it, it is necessary to improve the network substantially in order to obtain high real-time transmission. This problem is often divided into a distributed and a centralized solution. The distributed solution is based on the division of tasks by levels. This solution is very robust, but it lacks perspective. A centralized solution manages heterogeneous networks in a simple way, although the complexity is the weakness of this solution. 
Semantic technology is a relatively new technology from which much is expected. The main advantage of this technology is the sharing of knowledge through a wireless sensor network. Semantic representation and processing are very important for the development of intelligent Internet of Things systems. The main challenges of this field are the technology of semantic representation for terminal devices, query-based semantic platforms, and semantic analysis and management of sensor information.

\subsection{SECURITY CHALLENGES}

Security is one of the most important challenges in all technologies, so it is also important in wireless sensor networks. The data is often sensitive and of great importance. Unattended sensor nodes are much more sensitive to attacks. Wireless communication is also sensitive to attacks. There are many network defense techniques, but sensor networks do not have enough resources, which is why there is a problem with the implementation of defense. Some of the solutions are key distribution, cryptography, and node authentication [13]. Connecting wireless sensor networks to the Internet of Things increases security. A lot of people are entering the security of the Internet of Things, and thus into wireless sensor networks. There are many threats, and the damage they can cause is often enormous.

The biggest challenge of security is to make strong routing protocols. Such protocols can well protect the network from malicious attacks, and they can handle limited resources. It is necessary to design new and powerful protocols that will solve many security challenges. One solution is to use trust relations. If trust is high, there is little need for cryptographic keys. This way we save energy and network performances are improved.

\section{FIELDS OF RESEARCH}

There are many fields of research into the challenges and issues of wireless sensor networks, and there are fields that are still only in theory. Some of the challenges and issues we did not cover or mentioned are:

- Localization

- Middleware

- Synchronization

- Database Centric and Querying

- Calibration

- Geographic routing

- Programming Models

- Medium Access Schemes
One of the most interesting research fields is the use of wireless sensor networks in machine learning and artificial intelligence. Wireless sensor networks can be adapted to neural networks to save energy and facilitate tasks.

Another interesting research field is smart buildings; the basic challenges are energy and security. In addition to these challenges, there is also the cost of hardware, wireless connectivity, system architecture, and programmability.

Fields of research such as medicine, pharmacy, and biology are critical for human health. Therefore, the application of wireless sensor networks is of great importance for improving healthcare applications. These networks can accelerate the work of health care, and in the future may completely replace humans. To achieve this, it is necessary to improve challenges such as interoperability, real-time data acquisition and analysis, reliability and robustness, and new node architectures.

Agriculture is also a field in which the use of wireless sensor networks is becoming necessary. Monitoring the amount of water, soil quality, as well as the action of plant protection products is of great importance. That is why it is important to work on researching wireless network networks for this field.

\section{CONCLUSION}

The challenge area in wireless sensor networks is huge and not at all easy to handle. This paper summarizes all the major challenges in wireless sensor networks. Of course, numerous challenges exist, as so are not even mentioned. Challenge divisions were made based on network type, components, characteristics, Internet of Things, and security. It is possible to make more divisions, but here they are briefly summarized in this way. Several important challenges are mentioned in each section. Finally, there are a few words about the fields of research that are particularly interesting and important, as well as some indications of what will be worked on in the future. 


\section{REFERENCES}

[1] M. Zivkovic, R. Popovic and T. Tanaskovic, "The survey of commercially available sensor platforms from energy efficiency aspect," in 2012 20th Telecommunications Forum (TELFOR), Belgrade, Serbia, 2012.

[2] M. Zivkovic, I. Branovic, D. Markovic and R. Popovic, "Energy efficient security architecture for wireless sensor networks," in 2012 20th Telecommunications Forum (TELFOR), Belgrade, Serbia, 2012.

[3] E. Alaa, H. M. El-Bakry, S. M. A. Elrazik, S. Q. Hasan, A. Q. Hasan and S. Zaid, "Challenges in Wireless Sensor Networks," International Journal of Advanced Research in Computer Science \& Technology, vol. IV, no. 4, pp. 22-27, 2016.

[4] Y. A. Bangash, Q. u. D. Abid, A. Alshreef and Y. Alsalhi, "Security issues and challenges in Wireless Sensor Networks: A survey," IAENG International Journal of Computer Science, vol. XCIV, no. 2, pp. 135-149, 2017.

[5] Indu, "Wireless Sensor Networks: Issues \& Challenges," International Journal of Computer Science and Mobile Computing, vol. III, no. 6, pp. 681-685, 2014.

[6] K. Soundarapandian and A. Kumar, "Challenges of wireless sensor networks and issues associated with time synchronization," in UGC sponsored national conference on advanced networking and applications, Udumalpet, 2015.

[7] M. Elhoseny, J. Batle, A. Farouk, A. Shehab and A. E. Hassanien, "Secure Image Processing and Transmission Schema in Cluster-Based Wireless Sensor Network," in Handbook of Research on Machine Learning Innovations and Trends, IGI Global, 2017, pp. 1022-140.

[8] N. Bacanin, E. Tuba, M. Zivkovic, I. Strumberger and M. Tuba, "Whale Optimization Algorithm with Exploratory Move for Wireless Sensor Networks Localization," in International Conference on Hybrid Intelligent Systems, 2019.

[9] M. Zivkovic, N. Bacanin, E. Tuba, I. Strumberger, T. Bezdan and M. Tuba, "Wireless Sensor Networks Life Time Optimization Based on the Improved Firefly Algorithm," in 2020 International Wireless Communications and Mobile Computing (IWCMC), 2020.

[10] M. Zivkovic, T. Zivkovic, K. Venkatachalam and N. Bacanin, "Enhanced Dragonfly Algorithm Adapted for Wireless Sensor Network Lifetime Optimization," in Data Intelligence and Cognitive Informatics, 2021.
[11] M. Zivkovic, N. Bacanin, T. Zivkovic, I. Strumberger, E. Tuba and M. Tuba, "Enhanced Grey Wolf Algorithm for Energy Efficient Wireless Sensor Networks," in 2020 Zooming Innovation in Consumer Technologies Conference (ZINC), Novi Sad, Serbia, 2020.

[12] M. Živković and T. Živković, "Wireless Sensor Networks Integration Into Internet Of Things," in Sinteza 2017 - International Scientific Conference on Information Technology and Data Related Research, Belgrade, Serbia, 2017.

[13] M. Pule, R. Jamisola and F. Ibikunle, "Energy Requirements in Cryptographic Mechanisms for Secure Wireless Sensor Networks: An Overview," in International Conference on Clean Energy for Sustainable Growth In Developing Countries, Palapye, 2015. 